\title{
Giant Axial Dielectric Response in Water-Filled Nanotubes and Effective Electrostatic Ion-Ion Interactions from a Tensorial Dielectric Model
}

\author{
Philip Loche, $^{\dagger}$ Cihan Ayaz, ${ }^{\dagger}$ Alexander Schlaich, ${ }^{\ddagger}$ Yuki Uematsu, ${ }^{\mathbf{I}}, \S$ and \\ Roland R. Netz ${ }^{*, \dagger}$ \\ $\dagger$ Fachbereich Physik, Freie Universität Berlin, 14195 Berlin, Germany \\ $\ddagger$ Université Grenoble Alpes, CNRS, LIPhy, 38000 Grenoble, France \\ \Laboratoire de Physique de l'Ecole normale supérieure, ENS, Université PSL, CNRS, \\ Sorbonne Université, Université de Paris, F-75005 Paris, France \\ $\S$ Department of Physics, Kyushu University, 819-0395 Fukuoka, Japan \\ E-mail: rnetz@physik.fu-berlin.de
}

\begin{abstract}
Molecular dynamics simulations in conjunction with effective medium theory are used to investigate dielectric effects in water-filled nanotubes. The resulting effective axial dielectric constant shows a divergent increase for small nanotube radii that depends on the nanotube length, while the effective radial dielectric constant decreases significantly for thin nanotubes. By solving Poisson's equation for an anisotropic dielectric medium in cylindrical geometry, we show that the axial ion-ion interaction depends for small separations primarily on the radial dielectric constant, not on the axial one. This means that electrostatic ion-ion interactions in thin water-filled nanotubes are on the linear dielectric level significantly enhanced due to water confinement effects at small separations, while at large separations the outside medium dominates. If the outside medium is metallic, the ion-ion interaction decays exponentially for large ion separation.
\end{abstract}

\section{Introduction}

Aqueous pores have key functional roles in cells, membranes and proteins ${ }^{112}$, and are under intense investigation, for example in ion channels and photosynthesic complexes ${ }^{3 / 4}$. Additionally, they are interesting for technical applications such as water desalination ${ }^{516}$, signal multiplication $^{[7}$ and supercapacitors ${ }^{8[10}$ A simple model system for all these scenarios are water-filled carbon nanotubes (CNTs), which are studied intensively, both experimentally and in simulations ${ }^{11}[18$. In many applications, solvated ions are present in nanotubes and determine most of the salient CNT properties. Consequently, ionic transport $\frac{19}{21}$ and ionic conductivity ${ }^{2022[23]}$ of CNTs are at the focus of investigations. To describe the static and kinetic ion properties inside CNTs, the continuum PoissonBoltzmann and Helmholtz-Smoluchowski models are used $24 \sqrt[26]{26}$, but these models need as precise input predictions for the electrostatic ionion interactions, which in turn depend on the dielectric properties. The water dielectric response close to planar interfaces has been shown to be anisotropic and inhomogeneous ${ }^{27-29}$. In particular, the perpendicular water dielectric 
constant between two surfaces decreases significantly as the surface separation reaches the nanometer scale 30131 . Also for water confined inside CNTs the dielectric properties have been shown to differ from bulk water using simulations $^{32 / 33}$ as well as experiments ${ }^{34 / 35}$. However, to derive from dielectric properties the electrostatic interaction between ions requires the solution of the Poisson equation in the presence of tensorial and inhomogeneous dielectric profiles in cylindrical symmetry, which is far from trivial.

From molecular dynamics simulations of water inside single-walled armchair carbon nanotubes of various radii and lengths, we extract the tensorial dielectric radial and axial profiles. The definition of these profiles reflects the long range and non-locality of the dielectric response ${ }^{36137}$ and has been intensely discussed in literature 38 . With decreasing CNT radius, we find the axial dielectric response to be dramatically enhanced and to become CNT length dependent, which reflects the long-ranged dipolar ordering of water. The inverse radial dielectric response shows oscillations and multiple sign changes. For spatially varying dielectric tensorial profiles the electrostatic Green's function, i.e., the interaction between two point charges, cannot be calculated in closed form. We, therefore, use effective medium theory to extract effective axial and radial dielectric constants. These dielectric constants become anisotropic for nanotube radii $R<10$, nm, with the axial value diverging in a CNT-length dependent fashion and the radial value approaching the vacuum value in the single-file limit. By comparison with corresponding results for water in planar confinement, we show that the confinement effects in cylindrical confinement are even more drastic than in planar confinement, which suggests that curvature plays an important role in confinement. We also establish the Green's function formalism for tensorial electrostatics in cylindrical confinement. We find that for CNTs the axial ion-ion interaction is governed by the outside dielectric constant for large ionion distances, we present explicit results for the cases where the outside medium is dielectrically equivalent to vacuum and where the outside medium is metallic. For very small separations, the interaction only depends on the radial dielectric constant, not on the axial one, and thus is dramatically enhanced compared to the ionion interaction in bulk water. The axial ion-ion interaction in water-filled CNTs thus depends primarily on the radial dielectric water properties and exhibits complex crossovers. The most relevant message for experiments with ions in CNTs is the crossover due to the reduced radial dielectric component for small separation and the influence of the outside dielectric constant for large separations, which will influence ionic correlations and transport properties as well as chemical reactions $\underline{40}$.

\section{Methods}

\section{Theory}

For a cylindrical system that is homogenous along the axial coordinate $z$ and the angular coordinate $\varphi$, the $\varphi$ component of the rotation of the static electric field follows from Maxwell's equations as

$$
\frac{\partial E_{r}(r)}{\partial z}-\frac{\partial E_{z}(r)}{\partial r}=0 .
$$

Since the electric field is independent of $z$, the first term vanishes, and thus $E_{z}(r)=$ const. If no free charges are present, Gauss's law for the displacement field reads

$$
\frac{1}{r} \frac{\partial}{\partial r} r D_{r}(r)=0
$$

and thus $r D_{r}(r)=$ const. In linear response theory, the change of the displacement field, $\boldsymbol{D}(\boldsymbol{r})$, is related linearly to the change of the electric field, $\boldsymbol{E}(\boldsymbol{r})$, via the non local dielectric tensor $\hat{\varepsilon}_{\mathrm{nl}}\left(\boldsymbol{r}, \boldsymbol{r}^{\prime}\right)$ according to

$$
\boldsymbol{D}(\boldsymbol{r})=\varepsilon_{0} \int_{V} \hat{\varepsilon}_{\mathrm{nl}}\left(\boldsymbol{r}, \boldsymbol{r}^{\prime}\right) \boldsymbol{E}\left(\boldsymbol{r}^{\prime}\right) \mathrm{d} \boldsymbol{r}^{\prime},
$$

where $V$ is the system volume. Using the condition that $E_{z}(r)$ is constant, as follows from eq 
1. the $z$ component of eq 3 simplifies to

$$
D_{z}(r)=\varepsilon_{0} \varepsilon_{z}(r) E_{z}
$$

with $\varepsilon_{z}(r)=\int_{V} \varepsilon_{\mathrm{nl}}^{z z}\left(\boldsymbol{r}, \boldsymbol{r}^{\prime}\right) \mathrm{d} \boldsymbol{r}^{\prime}$. Similarly, the electric field can be written in terms of the change of the displacement field and the inverse dielectric response function as

$$
\boldsymbol{E}(\boldsymbol{r})=\varepsilon_{0}^{-1} \int_{V} \hat{\varepsilon}_{\mathrm{nl}}^{-1}\left(\boldsymbol{r}, \boldsymbol{r}^{\prime}\right) \boldsymbol{D}\left(\boldsymbol{r}^{\prime}\right) \mathrm{d} \boldsymbol{r}^{\prime} .
$$

Using the condition that $r D_{r}(r)$ is constant, as follows from eq 2 , the $r$ component of eq 5 is found to be

$$
E_{r}(r)=\varepsilon_{0}^{-1} \varepsilon_{r}^{-1}(r) D_{r}(r),
$$

where $\varepsilon_{r}^{-1}(r)=2 \pi r L \int_{0}^{\infty}\left[\varepsilon_{\mathrm{nl}}^{r r}\left(r, r^{\prime}\right)\right]^{-1} \mathrm{~d} r^{\prime}$. By inserting the relation

$$
\boldsymbol{D}(\boldsymbol{r})=\varepsilon_{0} \boldsymbol{E}(\boldsymbol{r})+\boldsymbol{m}(\boldsymbol{r})
$$

into eq 4, where $\boldsymbol{m}(\boldsymbol{r})$ denotes the difference of the polarization density with and without an applied external field, one obtains for the linear response axial dielectric profile

$$
\varepsilon_{z}(r)=1+\frac{m_{z}(r)}{\varepsilon_{0} E_{z}} \approx 1+\left.\frac{1}{\varepsilon_{0}} \frac{\partial m_{z}(r)}{\partial E_{z}}\right|_{E_{z}=0}
$$

where the response to the electric field is included to linear order by expanding $m_{z}(r)$. The linear response radial dielectric profile is obtained by substituting eq 7 into eq 6

$$
\varepsilon_{r}^{-1}(r)=1-\frac{m_{r}(r)}{D_{r}(r)} \approx 1-\left.r \frac{\partial m_{r}(r)}{\partial\left(r D_{r}(r)\right)}\right|_{r D_{r}(r)=0} .
$$

To obtain an expression for the polarization density derivative in eqs 8 and 9 , we use the canonical average

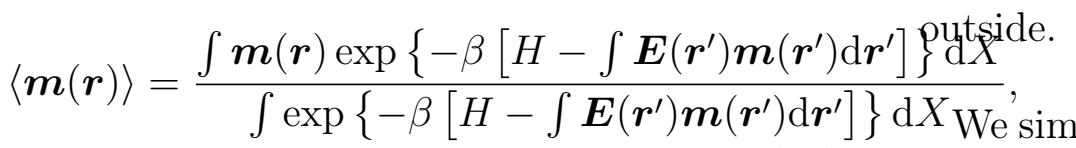

where $\beta$ is the inverse thermal energy and $H$ is the system Hamiltonian in the absence of an ex-

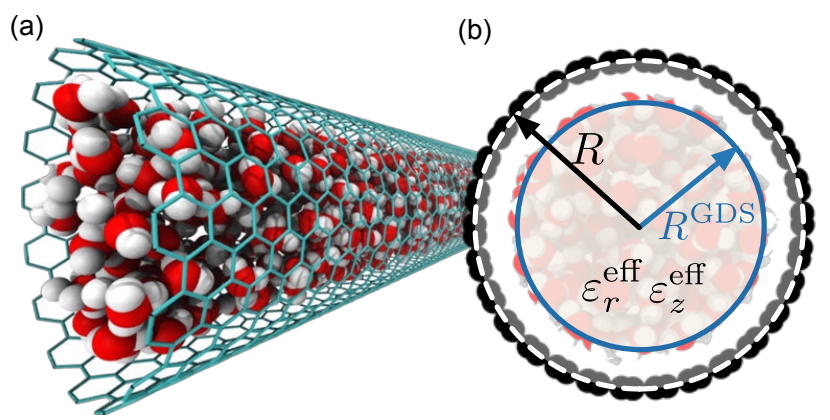

Figure 1: (a) Simulation snapshot of a waterfilled CNT with radius $R=1.2 \mathrm{~nm}$. (b) Representation of the effective medium model used for solving Poisson's equation. The permittivity is constant and anisotropic inside the Gibbs dividing radius $R^{\mathrm{GDS}}$ and isotropic and unity

ternal field. In this expression $\mathrm{d} X$ denotes the phase space integral over all positional degrees of freedom. Using eq 10 in eq 8 , we find for the axial dielectric profile

$$
\begin{aligned}
\varepsilon_{z}(r)=1 & +\frac{\beta 2 \pi L}{\varepsilon_{0}}\left(\left\langle m_{z}(r) \int_{0}^{\infty} m_{z}\left(r^{\prime}\right) r^{\prime} \mathrm{d} r^{\prime}\right\rangle\right. \\
& \left.-\left\langle m_{z}(r)\right\rangle\left\langle\int_{0}^{\infty} m_{z}\left(r^{\prime}\right) r^{\prime} \mathrm{d} r^{\prime}\right\rangle\right) .
\end{aligned}
$$

Similarly, by using eq 10 in eq 9 and employing eq 7. we obtain for the radial dielectric profile

$$
\begin{aligned}
\varepsilon_{r}^{-1}(r)=1 & -\frac{\beta 2 \pi r L}{\varepsilon_{0}}\left(\left\langle m_{r}(r) \int_{0}^{\infty} m_{r}\left(r^{\prime}\right) \mathrm{d} r^{\prime}\right\rangle\right. \\
& \left.-\left\langle m_{r}(r)\right\rangle\left\langle\int_{0}^{\infty} m_{r}\left(r^{\prime}\right) \mathrm{d} r^{\prime}\right\rangle\right) .
\end{aligned}
$$

Note that no locality assumptions are used to derive eqs 11 and 12 , which agree with previous results obtained by slightly different arguments 33 .

\section{Computational Details} lation box with periodic boundary conditions. The carbon atoms are frozen, unpolarizable and charge-neutral, for water we use the SPC/E 
model, see Figure 1 1 for a simulation snapshot. We define the CNT radius $R$ by the carbon atom position as given in eq $[S 1$ in the Supporting Information and shown in Figure $1 \mathrm{~b}$. For the carbon-oxygen Lennard-Jones interaction, we use the GROMOS53a6 force field ${ }^{41}$, as it accurately reproduces the water contact angle on carbon surfaces ${ }^{42}$. The dielectric profile at planar surfaces has been previously shown to depend slightly on the surface-water interaction strength $^{28}$, we expect a similar dependence also for the present cylindrical case, which however is not studied further. To determine the correct water density inside the CNTs, we first attach a reservoir to cylindrical tubes with a length of $L=15.1 \mathrm{~nm}$, from which we determine the water density in the central CNT section. The reservoir is pressure-coupled along the cylinder axes using an extended Hamiltonian and the Berendsen method, see section $\mathrm{S} 2$ of the Supporting Information for details. For the production runs, we use periodic CNTs without a reservoir in the NVT ensemble and the $\mathrm{v}$ rescale thermostat $\frac{43}{4}$ at $300 \mathrm{~K}$. Different temperature control methods have no effect on our results as shown in section $\mathrm{S} 3$ of the Supporting Information. Simulations were performed using the GROMACS simulation package 44 and we provide a free Python library (located at https://gitlab.com/netzlab/maicos) based on the MDAnalysis library ${ }^{45}$, which implements the analysis we used for extracting the dielectric profiles 28130139 . See sections S1 in the Supporting Information for further simulation details.

\section{Results and Discussion}

\section{Simulations}

From the MD simulations, the radial number density $n(r)$ and permittivity profiles $\varepsilon_{z}(r)$ and $\varepsilon_{r}^{-1}(r)$ are deduced according to eqs 11 and 12 and shown in Figure 2 for different $\mathrm{CNT}$ radii $R$. Profiles are for CNT length $L=15.1 \mathrm{~nm}$. Different lengths are shown in Figure S4 and S5 in section S4 of the Supporting Information. The density profiles $n(r)$ in Figure 2 a show the typical oscillations close to the CNT wall ${ }^{14}$.
The axial permittivity profiles $\varepsilon_{z}(r)$ in Figure $2 \mathrm{~b}$ are similar in shape to the density profiles, but for smaller radii $R$ the maxima in the $\varepsilon_{z}(r)$ profile become increasingly pronounced. The inverse radial dielectric profiles $\varepsilon_{r}^{-1}(r)$ in Figure 2 c are very different from $\varepsilon_{z}(r)$ and exhibit pronounced oscillations in the CNT center. To clarify the origin of these oscillations we extract $\varepsilon_{r}^{-1}(r)$ from simulations of a large homogeneous water box with periodic boundary conditions. The result, shown as a black dashed line in Figure 2k, perfectly superimposes on the data for $R=1.7 \mathrm{~nm}$ and $R=2.7 \mathrm{~nm}$. This shows that these oscillations are not caused by the presence of the CNT but rather are due to the water discreteness. In Figure $2 \mathrm{~d}-\mathrm{f}$, the profiles are shifted by the position of the nanotube radius $R$. It becomes clear that the oscillations in $n(r)$ and $\varepsilon_{z}(r)$ (and to a lesser degree also in $\varepsilon_{r}^{-1}(r)$ ) close to the CNT interface are rather independent of $R$ and agree quite nicely with the planar limit (solid black line), obtained from simulations of water between planar graphene sheets with a large separation. Only the axial dielectric profile for the narrowest CNT $(R=0.4 \mathrm{~nm})$ is length dependent, as shown in Figure $\mathrm{S} 4$ and discussed below.

\section{Effective Medium Theory}

In order to calculate ion-ion electrostatic interactions in CNTs analytically, the $r$-dependent dielectric tensorial profiles must be modeled by piecewise constant profiles using effective medium theory ${ }^{46}$, as schematically indicated in Figure 1 b. This construction is subtle and requires care, to make this mapping unique we require asymptotic integral invariances that are different for the axial and radial components. For the axial part we demand that the laterally averaged displacement field $\bar{D}_{z}$ in the $\mathrm{CNT}$ is the same for the actual dielectric profile $\varepsilon_{z}(r)$ and the effective piece-wise constant 

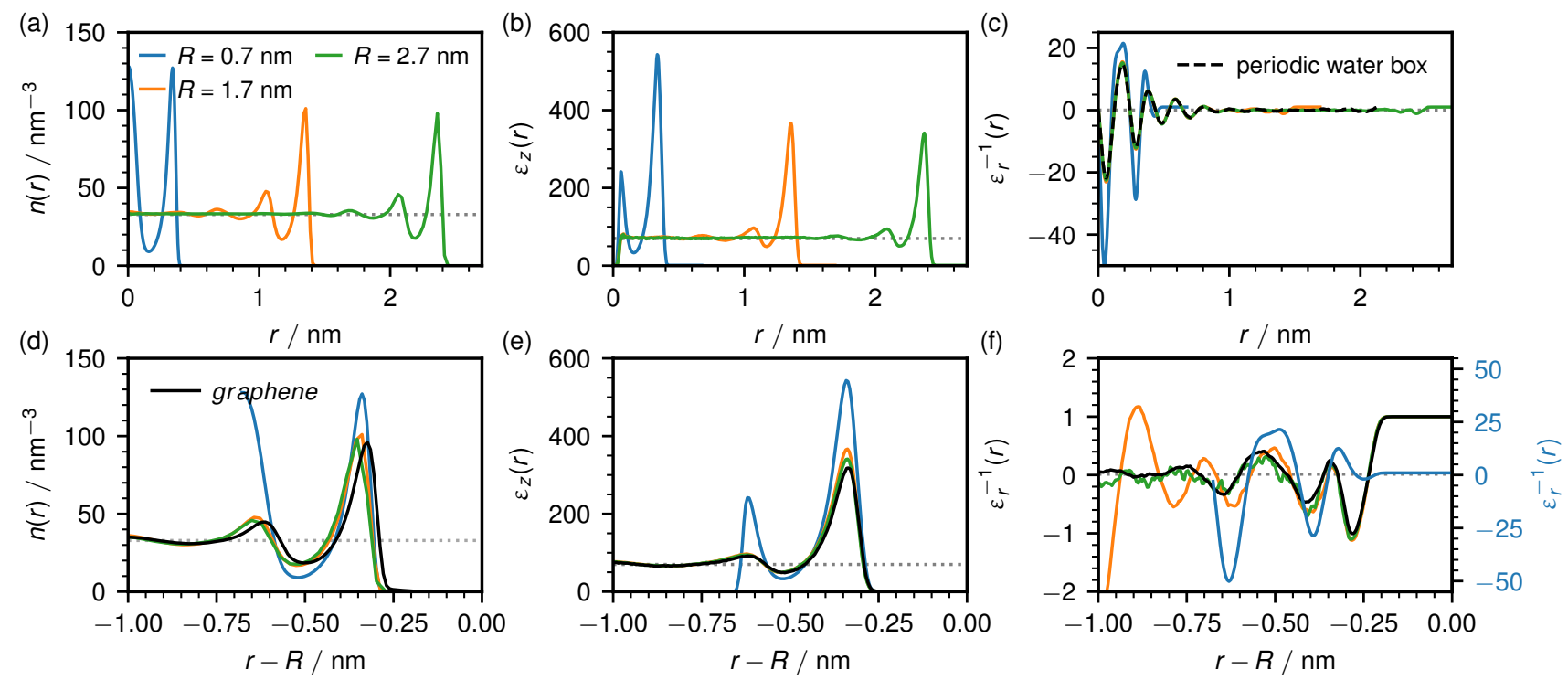

Figure 2: (a) Water oxygen number density profiles $n(r)$, (b) axial dielectric profiles $\varepsilon_{z}(r)$, (c) inverse radial dielectric profiles $\varepsilon_{r}^{-1}(r)$ for CNTs of several radii and fixed length $L=15.1 \mathrm{~nm}$. The horizontal broken lines depict the bulk values obtained from independent simulations. (d-e) The same results as in a-c, but shifted by the respective CNT radius $R$, together with the profile at a planar graphene interface, shown as a solid black line. The right axis in (f) applies to data for $R=0.7 \mathrm{~nm}$.

profile $\varepsilon_{z}^{*}(r)$. Using eq 4 we obtain

$$
\begin{aligned}
\bar{D}_{z} & =\frac{1}{\pi R^{2}} \int_{0}^{R} 2 \pi r D_{z}(r) \mathrm{d} r \\
& =\frac{2 E_{z} \varepsilon_{0}}{R^{2}} \int_{0}^{R} r \varepsilon_{z}(r) \mathrm{d} r \stackrel{!}{=} \frac{2 E_{z} \varepsilon_{0}}{R^{2}} \int_{0}^{R} r \varepsilon_{z}^{*}(r) \mathrm{d} r,
\end{aligned}
$$

where the integral ranges up to the CNT radius $R$. The effective profile $\varepsilon_{z}^{*}(r)$ is chosen to be a box profile with an effective dielectric constant of $\varepsilon_{z}^{\text {eff }}$,

$$
\varepsilon_{z}^{*}(r)=\left\{\begin{array}{ll}
\varepsilon_{z}^{\mathrm{eff}} & \text { if } r \leq R^{\mathrm{GDS}} \\
1 & \text { if } r>R^{\mathrm{GDS}}
\end{array} .\right.
$$

Substituting this into eq 13 , yields $\varepsilon_{z}^{\text {eff }}$ as

$$
\varepsilon_{z}^{\mathrm{eff}}-1=\frac{2 \int_{0}^{R} r\left(\varepsilon_{z}(r)-1\right) \mathrm{d} r}{\left(R^{\mathrm{GDS}}\right)^{2}} .
$$

The Gibbs dividing radius is $R^{\mathrm{GDS}}=$ $\sqrt{\left(N v_{w}\right) /(\pi L)}$, where $N$ is the number of water molecules and $v_{w}=0.0307 \mathrm{~nm}^{3}$ is the bulk molecular water volume. The result for $\varepsilon_{z}^{\text {eff }}$ is rather independent of our choice for the radius of the effective dielectric profile, as we show in section $\sqrt{\mathrm{S} 5}$ in the Supporting Information.

For the radial component, we demand the potential difference between inside and outside the CNT to be the same when using the actual dielectric profile $\varepsilon_{r}(r)$ and the effective box profile $\varepsilon_{r}^{*}(r)$. From the relation between the electrostatic potential $\Phi(r)$ and the electric field $E(r)$, $\partial \Phi(r) / \partial r=-E_{r}(r)$, using eq 6 and the fact that $r D_{r}(r)$ is constant, we obtain

$$
\begin{aligned}
\Phi(R)-\Phi\left(R_{0}\right) & =\varepsilon_{0}^{-1} r D_{r}(r) \int_{R_{0}}^{R} \frac{1}{r^{\prime} \varepsilon_{r}\left(r^{\prime}\right)} \mathrm{d} r^{\prime} \\
& \stackrel{!}{=} \varepsilon_{0}^{-1} r D_{r}(r) \int_{R_{0}}^{R} \frac{1}{r^{\prime} \varepsilon_{r}^{*}\left(r^{\prime}\right)} \mathrm{d} r^{\prime} .
\end{aligned}
$$

As for the axial component, $\varepsilon_{r}^{*}(r)$ is a box profile

$$
\frac{1}{\varepsilon_{r}^{*}(r)}=\left\{\begin{array}{ll}
1 / \varepsilon_{r}^{\mathrm{eff}} & \text { if } r \leq R^{\mathrm{GDS}} \\
1 & \text { if } r>R^{\mathrm{GDS}}
\end{array},\right.
$$

where $\varepsilon_{r}^{\text {eff }}$ is the effective radial dielectric con- 
stant. Substituting eq 17 into eq $16, \varepsilon_{r}^{\text {eff }}$ is determined as

$$
\frac{1}{\varepsilon_{r}^{\mathrm{eff}}}=\frac{\int_{R_{0}}^{R}\left(\varepsilon_{r}(r) r\right)^{-1} \mathrm{~d} r-\ln \left(R / R_{0}\right)}{\ln \left(R^{\mathrm{GDS}} / R_{0}\right)}
$$

The lower integration boundary $R_{0}$ in eq 18 corresponds to the radius at which the potential inside the CNT is determined. It is chosen such that $\varepsilon_{r}^{\text {eff }}$ for the largest radius $R=10.17 \mathrm{~nm}$ matches the SPC/E bulk permittivity of $\varepsilon=70$. There are several choices for $R_{0}$ that comply with this condition, we pick $R_{0}=0.38 \mathrm{~nm}$ for which $\varepsilon_{r}^{\text {eff }}$ grows monotonically with $R$ (details are given in section $S 6$ in the Supporting Information).

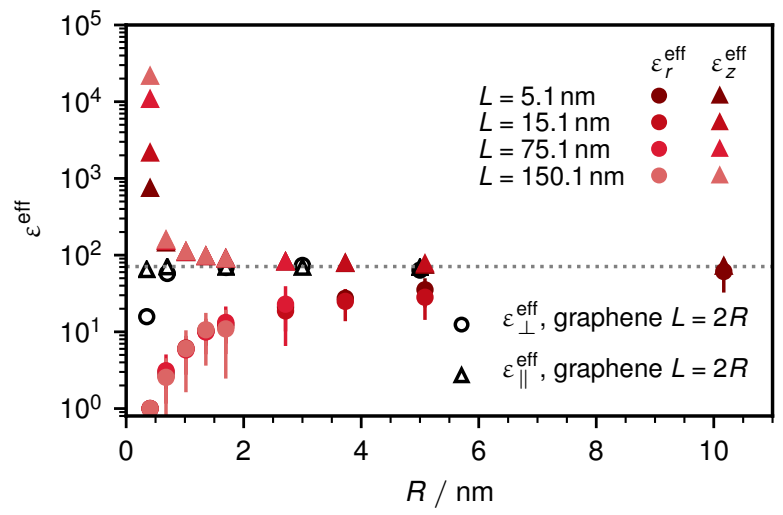

Figure 3: Effective radial (circles) and axial (triangles) dielectric water constants $\varepsilon_{r}^{\text {eff }}$ and $\varepsilon_{z}^{\text {eff }}$ for several CNT lengths and radii. The horizontal dotted line shows the bulk dielectric constant of SPC/E water $\varepsilon=70$. Open black circles and triangles show the effective perpendicular and parallel dielectric constants between planar graphene sheets at separation of $L=2 R$.

Figure 3 shows the effective dielectric constants $\varepsilon_{z}^{\text {eff }}$ and $\varepsilon_{r}^{\text {eff }}$ calculated according to eqs 15 and 18 . The radial part decreases whereas the axial part diverges as $R \rightarrow 0$. This behavior reflects the dipolar ordering of water molecules along the CNT axis, which becomes stronger with decreasing CNT radius, as will be explained by a simple Langevin model further below. For the smallest CNT radius, $R=0.4 \mathrm{~nm}$, $\varepsilon_{z}^{\text {eff }}$ in fact increases dramatically with increasing CNT length. This is caused by the increasing correlation length of the dipolar water or- dering and agrees nicely with analytical models for single-file water chains $\frac{47}{4}$, as we show in Figure S6 in the Supporting Information.

This anisotropic behavior of the dielectric behavior is somewhat similar to results for planar confinement ${ }^{30}$, where the dielectric component perpendicular to the surface was found to decrease and the parallel component was found to increase slightly with decreasing surface separation. To compare dielectric confinement effects in cylindrical to planar systems we calculate effective dielectric constants of water between two planar graphene sheets at a separation $L=2 R$ using the scheme introduced in Ref. 30. From the data shown in Figure 3, we conclude that the dielectric behavior of water in CNTs is rather different compared to water between planar graphene sheets and that confinement effects in cylinders are more pronounced than between planar sheets. This suggests that curvature effects play an important role in confinement. In fact, similar curvature effects were previously found also for the water friction coefficient in $\mathrm{CNTs}^{17}$. It is well known that the dielectric constant in bulk decreases significantly with rising temperature $\sqrt{48}$, an effect that is well reproduced in simulations ${ }^{49}$. In section S7 of the Supporting Information we show that the axial component of the effective dielectric constant decreases with temperature whereas the radial effective dielectric constant increases with temperature. Thus, the temperature dependence in cylindrical confinement differs from the bulk behavior, which is an interesting finding.

\section{Collective Versus Self Polarization}

In order to understand the cause of the pronounced difference between axial and radial dielectric effects, we investigate self and collective polarization fluctuations. For this we define the self and collective polarization variances as

$$
\begin{aligned}
C_{\alpha}^{\text {self }} & =\sum_{i}\left\langle p_{i, \alpha}^{2}\right\rangle-\left\langle p_{i, \alpha}\right\rangle^{2} \\
C_{\alpha}^{\text {coll }} & =\sum_{i} \sum_{j \neq i}\left\langle p_{i, \alpha} p_{j, \alpha}\right\rangle-\left\langle p_{i, \alpha}\right\rangle\left\langle p_{j, \alpha}\right\rangle,
\end{aligned}
$$




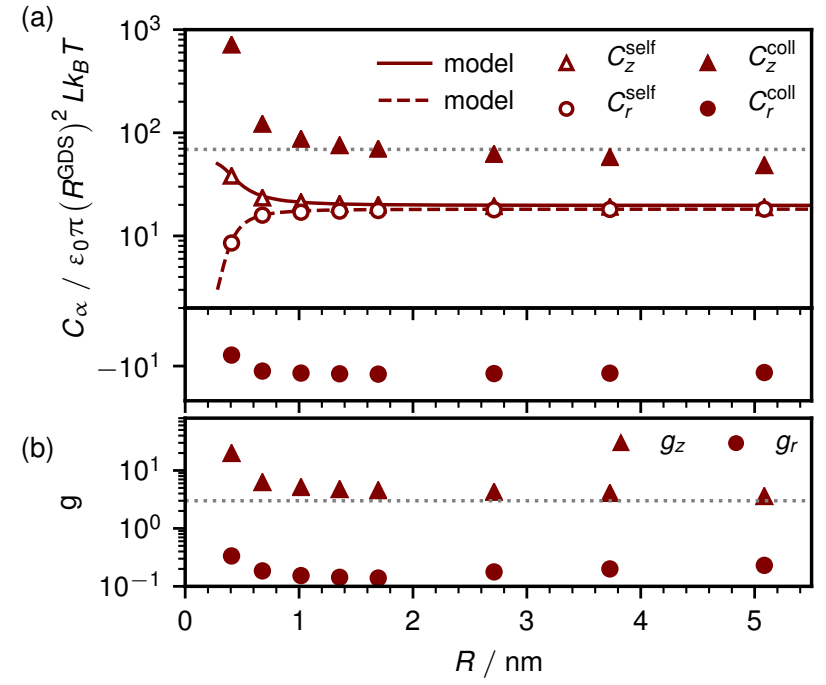

Figure 4: (a) Self and collective contributions of the rescaled polarization variances for axial (triangles) and radial (circles) directions. Solid and broken lines denote results from the anisotropic Langevin model eqs 24 and 25 for the self polarization variances. The horizontal dotted line shows the bulk dielectric constant of SPC/E water $\varepsilon-1=69$. (b) Kirkwood factors $g_{z}$ and $g_{r}$ obtained from axial and radial polarization fluctuations. The horizontal dotted line shows the bulk water Kirkwood factor $g=2.68^{50}$. where $p_{i, \alpha}$ is the dipole component $\alpha$ of molecule $i$ and $\alpha=x, y, z$. We define the average radial components as $C_{r}^{\text {self }}=$ $\left(C_{x}^{\text {self }}+C_{y}^{\text {self }}\right) / 2$ and $C_{r}^{\text {coll }}=\left(C_{x}^{\text {coll }}+C_{y}^{\text {coll }}\right) / 2$. The total polarization variance $C_{\alpha}$ is given by $C_{\alpha}=C_{\alpha}^{\text {self }}+C_{\alpha}^{\text {coll }}$, and in bulk the relation between $C_{\alpha}$ and the dielectric constant reads $\frac{37}{37}$

$$
\varepsilon_{\alpha}=1+\frac{C_{\alpha}}{\varepsilon_{0} V k_{B} T} .
$$

We note that there is a subtle difference between $C_{r}$ and the effective radial dielectric constant $\varepsilon_{r}^{\text {eff }}$, as an inspection of the defining eq 12 for the radial dielectric profile shows. Figure 4 a shows the polarization variances rescaled by $\varepsilon_{0} \pi\left(R^{\mathrm{GDS}}\right)^{2} L k_{B} T$, so that the data can be compared to the dielectric constants calculated earlier. For the axial part, collective contributions are positive and much larger than the self part. As shown in Figure $4 \mathrm{~b}$, the axial Kirkwood $g_{z}$ factor, defined as $g_{z}=C_{z} / C_{z}^{\text {self }}$, reaches for large CNT radii a value of $g_{z} \approx$ 3 , close to what is found in bulk water $\stackrel{50}{ }$, but for smaller radii drastically increases. For larger CNT radii therefore roughly three water molecules are axially correlated, while this number rapidly increases as the radius goes down. For the radial contribution, the behavior is completely different. Here, the collective contribution is negative, which means that the radial component of the orientation of adjacent waters is anti-correlated. We note that for very large radii the radial and axial collective contributions must become equal, but this crossover is very slow. In accordance with this, the radial Kirkwood factor $g_{r}=C_{r} / C_{r}^{\text {self }}$ in Figure $4 \mathrm{~b}$ is very small and approaches the bulk value very slowly.

\section{Anisotropic Langevin Model}

Here we generalize the Langevin model for the response of a single dipole to an applied electric field to account for anisotropic alignment effects. The Hamiltonian for the standard Langevin model reads $H=-\boldsymbol{p} \cdot \boldsymbol{E}$. We extend this by an biaxial alignment field with strength 
$c$ according to

$H=-p\left[E_{z} \cos (\vartheta)+E_{x} \sin (\vartheta) \cos (\varphi)\right]+c \cos (\vartheta)$,

which mimics the axial orientation of the water dipole along $z$ in narrow CNTs for $c<0$. We assume an external electric field in the $x-z$ plane and describe the dipole orientation by spherical coordinates with polar and azimuthal angles $\vartheta$ and $\varphi$. The permittivities are obtained from second derivatives of the partition function $Z$, the integral over phase space $Z=\int \mathrm{d} X \exp (-\beta H)$, with respect to the applied field $E$

$$
\varepsilon_{\alpha}=1+\frac{n}{\beta \varepsilon_{0}}\left[\frac{\partial}{\partial E_{\alpha}}\left(\frac{1}{Z} \frac{\partial Z}{\partial E_{\alpha}}\right)\right]_{E_{\alpha}=0},
$$

where $\alpha=z, x$. For the two components of the permittivity, as shown in section $[\mathrm{S} 8$ of the Supporting Information, we find

$$
\begin{aligned}
& \varepsilon_{z}=1+\frac{n p^{2}}{2 c \varepsilon_{0}}\left(1-\frac{2 \sqrt{\beta c} e^{-\beta c}}{\sqrt{\pi} \operatorname{erf}(\sqrt{\beta c})}\right) \\
& \varepsilon_{x}=1+\frac{n p^{2}}{4 c \varepsilon_{0}}\left(1-2 \beta c-\frac{2 \sqrt{\beta c} e^{-\beta c}}{\sqrt{\pi} \operatorname{erf}(\sqrt{\beta c})}\right),
\end{aligned}
$$

where $n$ is the number density and $\operatorname{erf}(x)$ the error function. In the limit of $c=0$ one recovers the standard Langevin model result $\varepsilon_{z}=\varepsilon_{x}=$ $1+\left(\beta n p^{2}\right) /\left(3 \varepsilon_{0}\right)$, which gives $\varepsilon_{z}=\varepsilon_{x}=19.71$ for a dipole moment $p=0.049 \mathrm{enm}$ and $n=$ $33.4 \mathrm{~nm}^{-3}$ for the SPC/E water model. Predictions for $\varepsilon_{z}-1$ (from eq 24) and $\varepsilon_{x}-1$ (from eq 25) are included in Figure $4 a$ as solid and broken lines and describe the self-polarization very well, where the radius-dependent alignment field strength $c(R)=c_{0}+b R^{-n}$ was fitted to the simulation data as shown in Figure S13 in the Supporting Information. The best fit is obtained for $c_{0}=-0.2 \mathrm{k}_{\mathrm{B}} \mathrm{T}, b=0.28 \mathrm{k}_{\mathrm{B}} \mathrm{T} \mathrm{nm}{ }^{n}$ and $n=2.8$. This shows that the symmetrybreaking between the axial and radial dielectric self responses can be described by an extended Langevin model that accounts for a radiusdependent axial water alignment.
Electrostatic Interactions in an Anisotropic Cylindrical Dielectric Medium

According to the effective medium model we derived from our simulation data, inside a cylinder of radius $R^{\mathrm{GDS}}$ the dielectric constants are anisotropic and given by $\varepsilon_{z}^{\text {eff }}$ and $\varepsilon_{r}^{\text {eff }}$, while outside the cylinder the dielectric constants are different and reflect the dielectric properties of the embedding medium, see Figure $1 \mathrm{~b}$. The electrostatic potential $\Phi(\boldsymbol{r})$ for an arbitrary charge distribution $\rho(\boldsymbol{r})$ follows from Poisson's equation

$$
\varepsilon_{0}\{\nabla \cdot[\hat{\boldsymbol{\varepsilon}}(\boldsymbol{r}) \cdot \nabla \Phi(\mathbf{r})]\}=-\rho(\boldsymbol{r}),
$$

where $\hat{\varepsilon}(\mathbf{r})$ is the anisotropic permittivity tensor. The derivation of $\Phi(\boldsymbol{r})$ for a point charge distribution $\rho(\boldsymbol{r})=q \delta\left(\boldsymbol{r}-\boldsymbol{r}^{\prime}\right)$ is explicitly shown in section S9 in the Supporting Information.

We consider the interaction energy $U(z)=$ $q^{\prime} \Phi(z)$ between two point charges that are located at the CNT axis at separation z. From the exact expression for $U(z)$, eq $\mathrm{S} 50$ in the Supporting Information, we find for small distances $z$ the asymptotic behavior

$$
\lim _{z \rightarrow 0} U(z)=\frac{q q^{\prime}}{4 \pi \varepsilon_{0} \varepsilon_{r}^{\mathrm{eff}}}\left[\frac{1}{z}+\frac{\alpha-1}{\alpha+1} \frac{\gamma R}{(z / 2)^{2}+(\gamma R)^{2}}\right],
$$

where $\alpha=\sqrt{\varepsilon_{z}^{\text {eff }} \varepsilon_{r}^{\text {eff }}}$ and $\gamma=\sqrt{\varepsilon_{z}^{\text {eff }} / \varepsilon_{r}^{\text {eff }}}$. For small $z$ the first term in eq 27 dominates and the interaction energy only depends on the effective radial dielectric constant $\varepsilon_{r}^{\text {eff }}$ but not on the axial one. As we show in section S9.1 in the Supporting Information, this at first sight surprising behavior follows quite generally for anisotropic homogeneous dielectric media and is not specific to the cylindrical geometry. In the limit $z \rightarrow \infty$ we find

$$
\lim _{z \rightarrow \infty} U(z)=\frac{q q^{\prime}}{4 \pi \varepsilon_{0} \varepsilon_{\text {out }}} \frac{1}{z} .
$$

As expected, for large $z$ the interaction energy only depends on the outer dielectric constant $\varepsilon_{\text {out }}$. For convenient use in coarse-grained sim- 
ulations we provide a simple heuristic crossover formula for $U(z)$ that exhibits the correct asymptotic behaviors in section S9.5 in the Supporting Information.

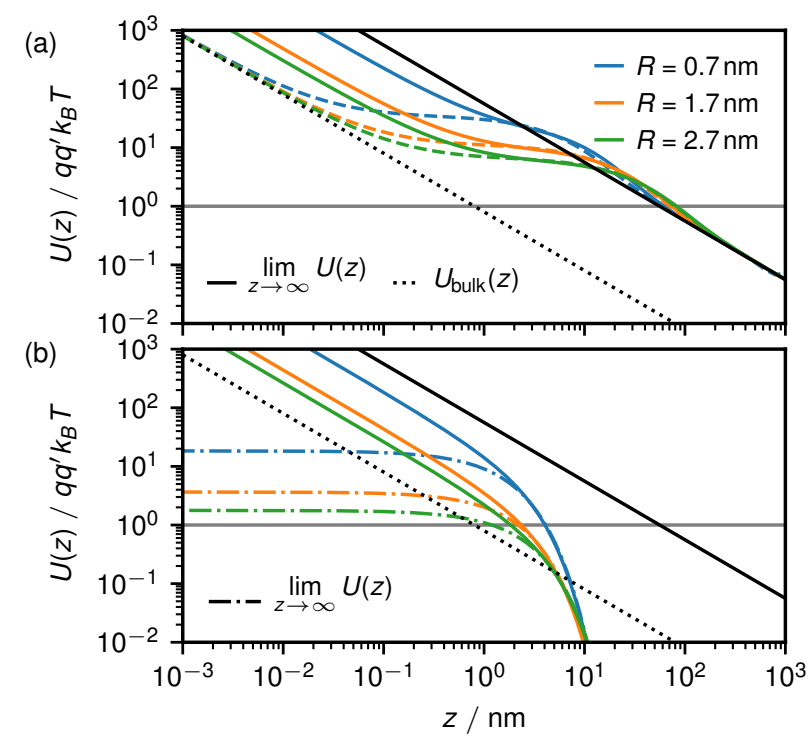

Figure 5: Electrostatic interaction energy $U(z)$ between two charges as a function of the distance $z$ along the axial direction inside a cylinder that is filled with an anisotropic dielectric medium, the outside medium in (a) is an insulator and modeled as vacuum and in (b) is a conductor and modeled as a metal. (a) Colored lines show $U(z)$ according to the exact solution of the Poisson equation, eq $\mathrm{S} 50$ in the Supporting Information. For the solid colored lines, we use the effective anisotropic dielectric constants as shown in Figure 3 . For the dashed colored lines we use isotropic dielectric constants of $\varepsilon_{r}^{\mathrm{eff}}=\varepsilon_{z}^{\mathrm{eff}}=70$. The dotted black line shows the ion-ion interaction in isotropic bulk water with $\varepsilon=70$, the solid black line shows the ionion interaction in vacuum with $\varepsilon=1$. (b) Solid lines show the exact solution inside a metallic medium according to eq S71 in the Supporting Information. The dashed dotted lines are the limiting result for large $z$ according to eq. 29 .

In Figure 57 we show results according to the exact expression for $U(z)$ given in eq $\mathbf{S 5 0}$ for the case where the outside medium is vacuum and thus $\varepsilon_{\text {out }}=0$; here we use the effective anisotropic dielectric constants inside the cylinder presented in Figure 3 for three differ- ent CNT radii as solid colored lines. For the dashed colored lines in Figure 5 a we also use eq $\mathrm{S} 50$ but rather take isotropic dielectric constants that correspond to $\mathrm{SPC} / \mathrm{E}$ bulk water $\varepsilon_{r}^{\text {eff }}=\varepsilon_{z}^{\text {eff }}=70$ inside the CNTs. The difference between these two predictions indicates that the interaction is in CNTs considerably enhanced below separations $z<2 \mathrm{~nm}$ due to the decrease of $\varepsilon_{r}^{\text {eff }}$ compared to bulk water. We also see that for distances $z>2 \mathrm{~nm}$ the interactions deviate from a pure power law $U(z) \sim 1 / z$ and thus are influenced by the outside medium. For large distances $z>10 \mathrm{~nm}$ the interactions only depend on the permittivity of the outside medium and approach eq 28, which is shown as a solid black line. The dotted black line shows the interaction in bulk water. We see that the interactions inside the CNTs are for an intermediate distance range $10 \mathrm{~nm}<z<100 \mathrm{~nm}$ even larger than in vacuum, which is caused by image charge effects. We conclude that the interactions between charges in CNTs are enhanced compared to bulk water because of the presence of a low-dielectric medium outside the $\mathrm{CNT}$ and because the radial effective dielectric constant is significantly reduced in small CNTs compared to bulk water.

The case where the outside medium is metallic is of considerable interest since carbon nanotubes can be conducting 51 . In Figure $5 \mathrm{~b}$ we present results for the limiting case where the outer dielectric constant diverges, $\varepsilon_{\text {out }}=\infty$, by solid lines. Due to the strong image charge effects, the interaction between charges becomes exponentially screened for large $z$ and decays as

$$
\lim _{z \rightarrow \infty} U(z)=\frac{q q^{\prime}}{\pi \varepsilon_{0} \alpha R} \frac{J_{2}\left(\xi_{1}\right)}{J_{2}\left(\xi_{1}\right)} e^{-\frac{\xi_{1} z}{\gamma R}},
$$

where $J_{n}(z)$ is the n-th order Bessel function of first kind and $\xi_{1} \approx 2.4048$ is the first zero of $J_{0}$. The exact solution of the metallic limit and the derivation of the limiting law is provided in section S9.6 of the Supporting Information.

The horizontal gray lines in Figures $5 \mathrm{a}$ and $\mathrm{b}$ denote the Bjerrum length $l_{B}$, which is the distance at which the electrostatic interaction between two unit charges equals $k_{B} T$. The Bjerrum length inside CNTs with diameters of the 
order of a few nanometers embedded in vacuum is seen to be about $100 \mathrm{~nm}$ and thus two orders of magnitude larger than in bulk water, where we have $l_{B} \approx 0.7 \mathrm{~nm}$. For metallic CNTs the Bjerrum length is about one order of magnitude larger compared to bulk water. This gives the electrostatic interaction in CNTs an enormous range that will dramatically enhance correlation effects.

\section{Conclusions}

We analyze the dielectric properties of waterfilled CNTs using MD simulations combined with effective medium theory. We find that the axial dielectric profile oscillates close to the CNT wall with an amplitude that depends on the CNT radius. This is due to water layering and matches well with the oscillations in the water density profiles. The behavior of the radial dielectric profiles is markedly different and does not reflect the water density profile. Effective medium theory is used to extract effective dielectric constants from these dielectric profiles. This, on the one hand, helps the interpretation of the dielectric effects and, on the other hand, enables us to calculate electrostatic interactions inside CNTs. We find that the effective axial dielectric constant dramatically increases with decreasing CNT radius, which by comparison with a simple extended Langevin model we trace back to axial water ordering in thin CNTs. The effective radial dielectric constant, in contrast, decreases with decreasing CNT radius.

By the exact solution of the anisotropic Poisson equation in cylindrical geometry, we demonstrate that the axial interaction between two ions inside a CNT depends for small separation primarily on the radial dielectric constant, not on the axial one. Thus, the ion-ion interaction is significantly enhanced for small separation due to the decrease of the radial dielectric constant for small $\mathrm{CNT}$ radii, compared to the hypothetical scenario where the dielectric constant would be isotropic and given by the bulk water value. If the outside medium has a low dielectric constant, the ion-ion interactions are determined by the outside medium for large separations and thus are enhanced compared to interactions in bulk water. If the outside medium is metallic the ion-ion interactions become exponentially screened for large separations.

Clearly, for ions of finite size, we expect deviations from the present calculation for point-like ions. In fact, extended charge distributions can be treated by Green's function methods that were used earlier by us for planar systems $s^{52}$. The effective anisotropic dielectric model for water inside CNTs developed in this paper will also be useful for future coarse-grained modeling of confined aqueous systems $s^{5354}$. In particular, the long-range nature of electrostatic ionion interactions in water-filled cylinders will be important to take into account in future modeling of ionic distributions, ionic conductivities and electrokinetic effects inside CNTs.

Acknowledgement Funding by the Deutsche Forschungsgemeinschaft (DFG) via grants SFB 1078 and SFB 1114 is acknowledged.

\section{Supporting Information Avail- able}

Simulation details, determination of the water density inside carbon nanotubes, dielectric profiles for different CNT lengths, comparison of the axial effective dielectric constant for singlefile water with an analytical model, thermostat and temperature effects on the dielectric constant, the effective dielectric constant for different effective radial and axial radii, results for different choices of the inner radius $R_{0}$, derivation of the extended Langevin model, derivation of the tensorial electrostatic model

\section{References}

(1) Sui, H.; Han, B.-G.; Lee, J. K.; Walian, P.; Jap, B. K. Structural basis of waterspecific transport through the AQP1 water channel. Nature 2001, 414, 872-878.

(2) Rasaiah, J. C.; Garde, S.; Hummer, G. 
Water in Nonpolar Confinement: From Nanotubes to Proteins and Beyond. Annu. Rev. Phys. Chem. 2008, 59, 713-740.

(3) Agre, P. The Aquaporin Water Channels. Proc Am Thorac Soc 2006, 3, 5-13.

(4) Pfeil, B. E.; Schoefs, B.; Spetea, C. Function and evolution of channels and transporters in photosynthetic membranes. Cell. Mol. Life Sci. 2014, 71, 979-998.

(5) Corry, B. Designing Carbon Nanotube Membranes for Efficient Water Desalination. J. Phys. Chem. B 2008, 112, 14271434.

(6) Shannon, M. A.; Bohn, P. W.; Elimelech, M.; Georgiadis, J. G.; Mariñas, B. J.; Mayes, A. M. Science and technology for water purification in the coming decades. Nature 2008, 452, 301-310.

(7) Tu, Y.; Xiu, P.; Wan, R.; Hu, J.; Zhou, R.; Fang, H. Water-mediated signal multiplication with Y-shaped carbon nanotubes. PNAS 2009, 106, 18120-18124.

(8) Frackowiak, E.; Jurewicz, K.; Delpeux, S.; Béguin, F. Nanotubular materials for supercapacitors. Journal of Power Sources 2001, 97-98, 822-825.

(9) Raymundo-Piñero, E.; Kierzek, K.; Machnikowski, J.; Béguin, F. Relationship between the nanoporous texture of activated carbons and their capacitance properties in different electrolytes. Carbon 2006, 44, 2498-2507.

(10) Feng, G.; Li, S.; Presser, V.; Cummings, P. T. Molecular Insights into Carbon Supercapacitors Based on RoomTemperature Ionic Liquids. J. Phys. Chem. Lett. 2013, 4, 3367-3376.

(11) Holt, J. K.; Park, H. G.; Wang, Y.; Stadermann, M.; Artyukhin, A. B.; Grigoropoulos, C. P.; Noy, A.; Bakajin, O. Fast Mass Transport Through Sub-2Nanometer Carbon Nanotubes. Science 2006, 312, 1034-1037.
(12) Majumder, M.; Chopra, N.; Andrews, R.; Hinds, B. J. Nanoscale hydrodynamics: Enhanced flow in carbon nanotubes. $\mathrm{Na}$ ture 2005, 438, 44.

(13) Whitby, M.; Cagnon, L.; Thanou, M.; Quirke, N. Enhanced Fluid Flow through Nanoscale Carbon Pipes. Nano Lett. 2008, 8, 2632-2637.

(14) Alexiadis, A.; Kassinos, S. Molecular Simulation of Water in Carbon Nanotubes. Chem. Rev. 2008, 108, 5014-5034.

(15) Hummer, G.; Rasaiah, J. C.; Noworyta, J. P. Water conduction through the hydrophobic channel of a carbon nanotube. Nature 2001, 414, 188-190.

(16) Mukherjee, B.; Maiti, P. K.; Dasgupta, C.; Sood, A. K. Single-File Diffusion of Water Inside Narrow Carbon Nanorings. ACS Nano 2010, 4, 985-991.

(17) Falk, K.; Sedlmeier, F.; Joly, L.; Netz, R. R.; Bocquet, L. Molecular Origin of Fast Water Transport in Carbon Nanotube Membranes: Superlubricity versus Curvature Dependent Friction. Nano Lett. 2010, 10, 4067-4073.

(18) Chakraborty, S.; Kumar, H.; Dasgupta, C.; Maiti, P. K. Confined Water: Structure, Dynamics, and Thermodynamics. Acc. Chem. Res. 2017, 50, 2139-2146.

(19) Samoylova, O. N.; Calixte, E. I.; Shuford, K. L. Molecular Dynamics Simulations of Ion Transport in Carbon Nanotube Channels. J. Phys. Chem. C 2015, 119, 1659-1666.

(20) Secchi, E.; Niguès, A.; Jubin, L.; Siria, A.; Bocquet, L. Scaling Behavior for Ionic Transport and its Fluctuations in Individual Carbon Nanotubes. Phys. Rev. Lett. 2016, 116, 154501.

(21) Secchi, E.; Marbach, S.; Niguès, A.; Stein, D.; Siria, A.; Bocquet, L. Massive radius-dependent flow slippage in carbon nanotubes. Nature 2016, 537, 210-213. 
(22) Zwolak, M.; Wilson, J.; Ventra, M. D. Dehydration and ionic conductance quantization in nanopores. J. Phys.: Condens. Matter 2010, 22, 454126.

(23) Kavokine, N.; Marbach, S.; Siria, A.; Bocquet, L. Ionic Coulomb blockade as a fractional Wien effect. Nat. Nanotechnol. 2019, 14, 573-578.

(24) Schoch, R. B.; Han, J.; Renaud, P. Transport phenomena in nanofluidics. Rev. Mod. Phys. 2008, 80, 839-883.

(25) Bocquet, L.; Charlaix, E. Nanofluidics, from bulk to interfaces. 2010, 39, 10731095 .

(26) Bonthuis, D. J.; Netz, R. R. Unraveling the Combined Effects of Dielectric and Viscosity Profiles on Surface Capacitance, Electro-Osmotic Mobility, and Electric Surface Conductivity. Langmuir 2012, 28, 16049-16059.

(27) Stern, H. A.; Feller, S. E. Calculation of the dielectric permittivity profile for a nonuniform system: Application to a lipid bilayer simulation. J. Chem. Phys. 2003, 118, 3401-3412.

(28) Bonthuis, D. J.; Gekle, S.; Netz, R. R. Profile of the static permittivity tensor of water at interfaces: consequences for capacitance, hydration interaction and ion adsorption. Langmuir 2012, 28, 76797694 .

(29) Zhang, C.; Gygi, F.; Galli, G. Strongly Anisotropic Dielectric Relaxation of Water at the Nanoscale. J. Phys. Chem. Lett. 2013, 4, 2477-2481.

(30) Schlaich, A.; Knapp, E. W.; Netz, R. R. Water Dielectric Effects in Planar Confinement. Phys. Rev. Lett. 2016, 117, 048001 .

(31) Fumagalli, L.; Esfandiar, A.; Fabregas, R.; Hu, S.; Ares, P.; Janardanan, A.; Yang, Q.; Radha, B.; Taniguchi, T.;
Watanabe, K. et al. Anomalously low dielectric constant of confined water. Science 2018, 360, 1339-1342.

(32) Mikami, F.; Matsuda, K.; Kataura, H.; Maniwa, Y. Dielectric Properties of Water inside Single-Walled Carbon Nanotubes. ACS Nano 2009, 3, 1279-1287.

(33) Schaaf, C.; Gekle, S. Spatially resolved dielectric constant of confined water and its connection to the non-local nature of bulk water. The Journal of Chemical Physics 2016, 145, 084901.

(34) Cui, H.-B.; Takahashi, K.; Okano, Y.; Kobayashi, H.; Wang, Z.; Kobayashi, A. Dielectric Properties of Porous Molecular Crystals That Contain Polar Molecules. Angewandte Chemie International Edition 2005, 44, 6508-6512.

(35) Zhou, B.; Kobayashi, A.; Cui, H.-B.; Long, L.-S.; Fujimori, H.; Kobayashi, H. Anomalous Dielectric Behavior and Thermal Motion of Water Molecules Confined in Channels of Porous Coordination Polymer Crystals. J. Am. Chem. Soc. 2011, 133, 5736-5739.

(36) Fröhlich, H. Theory of Dielectrics: Dielectric Constant and Dielectric Loss; Oxford University Press, 1987.

(37) Neumann, M. Dipole moment fluctuation formulas in computer simulations of polar systems. Molecular Physics 1983, 50, 841-858.

(38) Gekle, S.; Arnold, A. Comment on "Anomalous Dielectric Behavior of Nanoconfined Electrolytic Solutions". Phys. Rev. Lett. 2013, 111, 089801.

(39) Loche, P.; Wolde-Kidan, A.; Schlaich, A.; Bonthuis, D. J.; Netz, R. R. Comment on "Hydrophobic Surface Enhances Electrostatic Interaction in Water". Phys. Rev. Lett. 2019, 123, 049601.

(40) Muñoz-Santiburcio, D.; Marx, D. Chemistry in nanoconfined water. Chem. Sci. 2017, 8, 3444-3452. 
(41) Oostenbrink, C.; Villa, A.; Mark, A. E.; Gunsteren, W. F. V. A biomolecular force field based on the free enthalpy of hydration and solvation: The GROMOS forcefield parameter sets 53A5 and 53A6. Journal of Computational Chemistry 2004, 25, 1656-1676.

(42) Werder, T.; Walther, J. H.; Jaffe, R. L.; Halicioglu, T.; Koumoutsakos, P. On the Water-Carbon Interaction for Use in Molecular Dynamics Simulations of Graphite and Carbon Nanotubes. J. Phys. Chem. B 2003, 10\%, 1345-1352.

(43) Bussi, G.; Donadio, D.; Parrinello, M. Canonical sampling through velocity rescaling. The Journal of Chemical Physics 2007, 126, 014101.

(44) Abraham, M. J.; Murtola, T.; Schulz, R.; Páll, S.; Smith, J. C.; Hess, B.; Lindahl, E. GROMACS: High performance molecular simulations through multi-level parallelism from laptops to supercomputers. SoftwareX 2015, 1-2, 19-25.

(45) Michaud-Agrawal, N.; Denning, E. J.; Woolf, T. B.; Beckstein, O. MDAnalysis: A toolkit for the analysis of molecular dynamics simulations. Journal of Computational Chemistry 2011, 32, 2319-2327.

(46) Hasted, J. B. Aqueous Dielectrics (Studies in Chemical Physics); Chapman and Hall, 1974 .

(47) Köfinger, J.; Dellago, C. Orientational Dynamics and Dielectric Response of Nanopore Water. Phys. Rev. Lett. 2009, 103, 080601.

(48) Israelachvili, J. N. Intermolecular and Surface Forces, 3rd ed.; Elsevier Science, 2010.

(49) Sedlmeier, F.; Netz, R. R. Solvation thermodynamics and heat capacity of polar and charged solutes in water. The Journal of Chemical Physics 2013, 138.
(50) Oster, G.; Kirkwood, J. G. The Influence of Hindered Molecular Rotation on the Dielectric Constants of Water, Alcohols, and Other Polar Liquids. J. Chem. Phys. 1943, 11, 175-178.

(51) Dresselhaus, M.; Dresselhaus, G.; Saito, R. Physics of carbon nanotubes. Carbon 1995, 33, 883-891.

(52) Loche, P.; Ayaz, C.; Schlaich, A.; Bonthuis, D. J.; Netz, R. R. Breakdown of Linear Dielectric Theory for the Interaction between Hydrated Ions and Graphene. J. Phys. Chem. Lett. 2018, 9, 6463-6468.

(53) Maggs, A. C.; Everaers, R. Simulating Nanoscale Dielectric Response. Phys. Rev. Lett. 2006, 96, 230603.

(54) Simoncelli, M.; Ganfoud, N.; Sene, A.; Haefele, M.; Daffos, B.; Taberna, P.L.; Salanne, M.; Simon, P.; Rotenberg, B. Blue Energy and Desalination with Nanoporous Carbon Electrodes: Capacitance from Molecular Simulations to Continuous Models. Phys. Rev. X 2018, 8, 021024 . 
Graphical TOC Entry

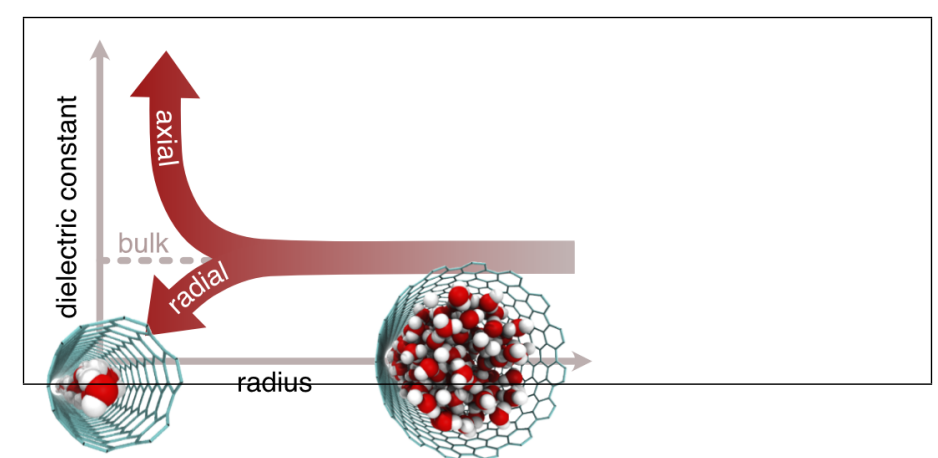

